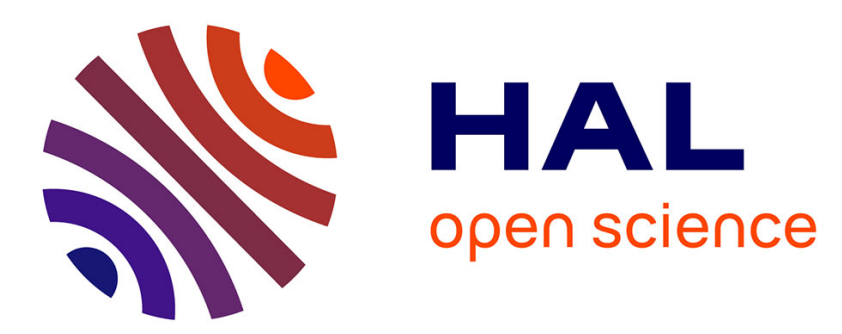

\title{
Analysis of the Stray Magnetic Field Created by Faulty Electrical Machines
}

Viet Phuong Bui, Olivier Chadebec, Laure-Line Rouve, Jean-Louis Coulomb

\section{To cite this version:}

Viet Phuong Bui, Olivier Chadebec, Laure-Line Rouve, Jean-Louis Coulomb. Analysis of the Stray Magnetic Field Created by Faulty Electrical Machines. COMPEL: The International Journal for Computation and Mathematics in Electrical and Electronic Engineering, 2008, 27 (1), pp.224-234. 10.1108/03321640810836780 . hal-03047924

\section{HAL Id: hal-03047924 https://hal.science/hal-03047924}

Submitted on 9 Dec 2020

HAL is a multi-disciplinary open access archive for the deposit and dissemination of scientific research documents, whether they are published or not. The documents may come from teaching and research institutions in France or abroad, or from public or private research centers.
L'archive ouverte pluridisciplinaire HAL, est destinée au dépôt et à la diffusion de documents scientifiques de niveau recherche, publiés ou non, émanant des établissements d'enseignement et de recherche français ou étrangers, des laboratoires publics ou privés. 


\title{
Analysis of the stray magnetic field created by faulty electrical machines
}

\author{
V.P. Bui, O. Chadebec, L-L. Rouve and J-L. Coulomb \\ Grenoble Electrical Engineering Laboratory, Domaine Universitaire, \\ Saint Martin d'Hères, France
}

\begin{abstract}
Purpose - This paper aims to compute the magnetic stray field created by faulty electrical machines. Design/methodology/approach - This paper proposes two approaches to compute the magnetic stray field created by faulty electrical machines. The first one presents a homogenized FEM method. The second one is based on a combination of an analytical expression for the magnetic field in the machine air gap with an integral method.
\end{abstract}

Findings - The studies show good agreement and demonstrate the reliability of the approach.

Originality/value - Two models developed in this paper originally used to compute the stray magnetic field of electrical machines. They can contribute to develop new tools for fault monitoring.

Keywords Electric machines, Finite element analysis, Electrical faults, Magnetic fields, Measurement

Paper type Technical paper

\section{Introduction}

The analysis of stray magnetic field created by electrical machines is known as a method for fault monitoring (Selvaggi et al., 2004; Henao et al., 2003). However, this task is not without complexity, the main difficulties being the need of a 3D model for computing the stray magnetic field and a high ratio between the internal and the external magnetic fields $\left(10^{6}\right)$.

An accurate formulation to compute the stray field has been proposed (Froidurot et al., 2002). It is based on a standard FEM computation with infinite box and then a post-processing with magnetic moments method. A first magnetostatic scalar FEM resolution is achieved. Once the scalar potential is obtained in the whole machine, the magnetization $\mathbf{M}$ in ferromagnetic parts $V_{\text {mag }}$ is computed. Then it is possible to compute the stray field thanks to a volume integral equation which links the magnetization $\mathbf{M}$ to the outside magnetic induction $\mathbf{B}$ :

$$
\overrightarrow{\mathbf{B}}=\frac{\mu_{0}}{4 \pi} \iiint_{V_{\text {mag }}}\left(3 \frac{\overrightarrow{\mathbf{M}} \cdot \vec{r}}{r^{5}} \vec{r}-\frac{\overrightarrow{\mathbf{M}}}{r^{3}}\right) \mathrm{d} V+\vec{B}_{0}
$$

where $r$ is the distance between the point where the magnetic induction is computed and the integration point, $\mathbf{B}_{0}$ is the induction created by winding. This integral post-processing approach is called moment method has been successfully validated by measurements (Froidurot et al., 2002).

However, this 3D modeling is very memory consuming. In the case of faulty machines, geometry or physical symmetries are lost. Thus, the number of unknowns becomes too much significant to allow the modeling of the whole machine. 
Therefore, the goal of this paper is to propose new models to evaluate the influence of faulty operation modes on the stray magnetic field of a synchronous machine, while keeping an acceptable number of unknowns. The first one carried out with homogenized FEM approach. This method is already known, but is originally adapted to the stray magnetic field computation. The second one is an original use of a classical integral method combined with an analytical expression of the induction in the machine air gap.

\section{Proposed models}

\subsection{Homogenized FEM model}

The basic idea of this model is to replace the tooth and slot area by an equivalent homogenized continuous materials with characteristic $\mathbf{B}(\mathbf{H})$.

The main assumption lies in an idealized distribution of induction flux inside the machine. The fields lines are tangential (according to a circular way) in the yoke and become radial in a zone located in the vicinity of the air gap and delimited, on both sides, by the end of the slots of the stator and the rotor. In the absence of a significant induced reaction (during no-load operation mode), this assumption is realistic because it does not modify the total reluctance associated inductor flux. Another assumption of our model concerns the linear materials law.

To schematize the behavior of the induction flux in teeth, let us consider the Figure 1, in which $\mathbf{B}_{t}$ and $\mathbf{B}_{s}$ are average induction values, respectively, in the teeth and the slots.

Let us suppose that the average values of the excitation field in the iron and in the vacuum are both equals to the given value $\mathbf{H}$. The average induction $\mathbf{B}$ on this tooth and slot area is thus given by the expression:

$$
\mathbf{B}(\mathbf{H})=\frac{\mu_{0} \times \mu_{r} \times H \times L_{t}+\mu_{0} \times H \times L_{s}}{L_{t}+L_{s}}
$$

where $\mu_{r}$ is the permeability of the ferromagnetic material, $L_{t}$ and $L_{s}$ present the widths of tooth and slot.

Thus, equation (2) leads to a homogenized equivalent permeability for each tooth and slot area. Moreover, as the magnetic flux density is mainly forced to remain radial, the permeability is then anisotropic. The radial $\mu_{1}$, tangential $\mu_{2}$, vertical $\mu_{3}$ components of this permeability are calculated by:

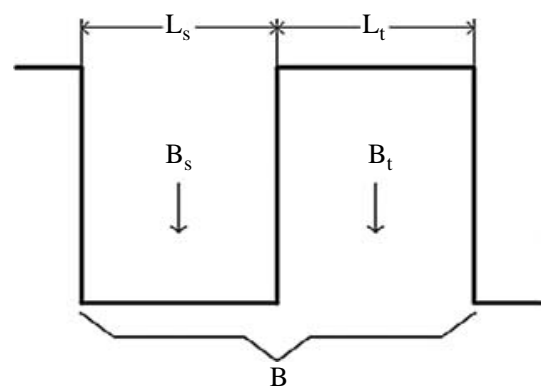

Figure 1. Simplified tooth and slot, associated induction in the electric machine 


$$
\begin{gathered}
\mu_{1}=\mu_{3}=\frac{\mu_{r} \times L_{t}+L_{s}}{L_{t}+L_{s}} \\
\mu_{2}=\frac{L_{t}+L_{s}}{L_{s}}
\end{gathered}
$$

As the consequence, we only need a coarse mesh in each tooth and slot area, in comparison with standard FEM modeling where to take into account the real slot geometry leads to a very fine mesh. The number of unknowns is largely decreased, which allows modeling the complete machine without symmetry. In particular, several fault operation modes can be modeled (rotor short-circuits, for example).

The stray magnetic field is computed by a post-processing with magnetic moments method.

However, this kind of 3D FEM modeling is still difficult and heavy to manage (geometry description and mesh, need of CPU memory, problem of cancellation error). This is why, a simpler model is proposed below.

\subsection{Air gap surface model}

This model is based on a combination between an analytical expression of the magnetic field in the machine air gap and an integral method.

In a first assumption, we consider that the materials law is linear and the slot geometry is not taken into account. The model is then carried out by considering two remarks as follows.

2.2.1 Field due to magnetizations. Denote $V$ a ferromagnetic body that channels a flux of induction (high-magnetic permeability). The field created by this device can be computed by the well known expression:

$$
\overrightarrow{\mathbf{B}}(x)=\mu_{0} \iiint_{V} g\left(\overrightarrow{\mathbf{M}}\left(x^{\prime}\right)\right) \mathrm{d} V
$$

where $x$ is the measurement point, $x^{\prime}$ is the integration point, $g$ is the mathematical expression of the field created by a dipole.

In such a case, it is usual to consider that in this volume, $\mathbf{M}$ has a free divergence. The volume $V$ creates exactly the same field as a surface $S$ with a charge distribution $q=\mathbf{M} \cdot \mathbf{n}$, where $\mathbf{n}$ is the normal going outside from the volume. More precise explanations of this representation can be found (Durand, 1968). Then, the field can be computed by:

$$
\overrightarrow{\mathbf{B}}(x)=\mu_{0} \iint_{S} f\left(\overrightarrow{\mathbf{M}}\left(x^{\prime}\right) \cdot \overrightarrow{\mathbf{n}}\left(x^{\prime}\right)\right) \mathrm{d} S
$$

where $f$ is the mathematical expression of the field created by a charge. This approach is called Coulombian representation of a ferromagnetic body.

In the example shown in Figure 2, volume $V$ channels perfectly the induction flux. Charges located on all the faces where $\mathbf{M}$ is perpendicular to the normal vanish. Only two opposite charges remain at the top and bottom of the volume.

2.2.2 Field due to the windings. Let us now consider a single loop $C$ in which flows a current $I$. The magnetic field created by this current is the same as the one created by 
every surface $S$ delimited by $C$ with a dipole distribution equal to $I \cdot \mathbf{n}$, where $\mathbf{n}$ is the normal to the surface (Durand, 1968). It is easy to see that such a surface dipole distribution is equivalent to the line current. To this end, we consider a network of small loop currents. The contributions of all currents cancel each other except on the line $C$. If the sizes of the small loops tend to 0 , we get a distribution of current vortex equivalent to dipoles. This result is known as the Ampere equivalence (Figure 3).

Let us now consider an electrical machine made of windings, an air gap, a stator and rotor. Under the equivalences above, the stray magnetic field is created (Durand, 1968):

- inductor coils in which currents flow; and

- charge surface density $\mathbf{M} \cdot \mathbf{n}$ located on surfaces which delimits the interface between ferromagnetic materials and air regions ( $\mathbf{M}$ is the magnetization of the materials and $\mathbf{n}$ the external normal going from the materials to the air).

In an electrical machine, three main surfaces must be considered. The first one is the external boundary (stator/air interface), and the two others deals with the air gap (air gap/stator and air gap/rotor) which are closed to each other. These two last surfaces carry nearly same charges distribution, opposite signs (the induction in the air gap is mainly radial and continuous so associated magnetizations are the same and both normal have opposite directions). If we neglect the slot geometry, both surfaces can be replaced by an average one of the air gap carrying a distribution of normal dipoles $T_{\text {mag. }}$.

In most part of electrical machine, this dipole distribution is easy to determine, the field $\mathbf{B}$ in the air gap being well known and directly linked to $\mathbf{M}$. Many analytical expressions already exist which depend, for example, on the poles number and the induction in the air gap. So, for a $2 p$-pole electrical machine with a sinusoidal air gap induction, this distribution created by the ferromagnetic materials can be represented:
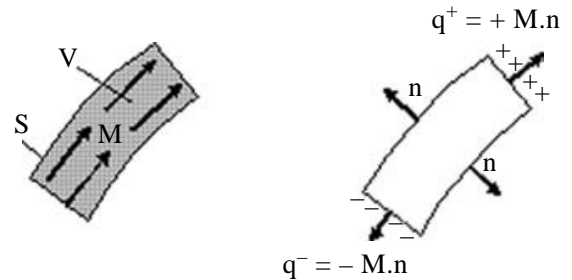

Note: Both magnetic fields computed are the same

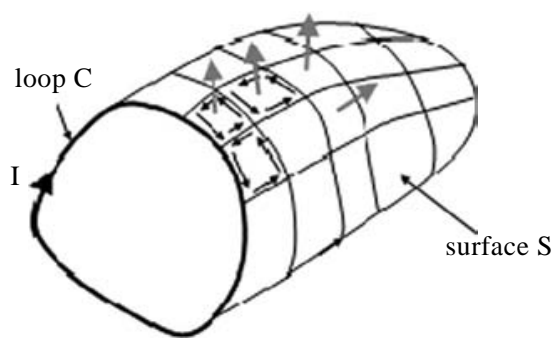

Figure 2.

Representation of a magnetized volume and its equivalent surface charge distribution
Figure 3. Equivalence between the current flowing in a winding and a distribution of normal dipoles 


$$
T_{\text {mag }}(\theta, t)=e \times \frac{\mu_{r}-1}{\mu_{0} \mu_{r}} \times B_{\text {airgap } \_ \text {max }} \times \cos \left(p \theta-\omega_{s} t\right)
$$

where $e$ is the thickness of the air gap, $\mu_{r}$ is the relative permeability of the material, $\theta$ is the mechanical position of the rotor and $\omega_{s}$ is the electrical pulsation and $t$ is the time.

We can now apply the equivalence with rotor windings, lines $C$ being the coils and the surface $S$ being the average surface of the air gap. In most part of an electrical machine, the sum of the different $I \cdot \mathbf{n}$ dipoles created by all the windings can be considered, in a first approximation, as sinusoidal. So, the field created by the windings can be represented by a distribution of normal dipoles located on the cylindrical average air gap surface. Its expression is:

$$
T_{\text {wind }}(\theta, t)=-A_{t_{-} \max } \times \cos \left(p \theta-\omega_{s} t\right)
$$

where $A_{t \_ \text {max }}$ is the maximum number of Ampere-turn for one pole. Let us notice that with such a distribution, end-winding effect is taken into account in the model.

Thus, a distribution of normal dipoles $T$ is obtained on the cylindrical surface in the air gap which is the sum of $T_{\text {mag }}$ or $T_{\text {wind }}$ and takes into account both ferromagnetic and windings effects. Note that $T_{\text {wind }}$ and $T_{\text {mag }}$ have opposite directions. In others words, $T_{\text {wind }}$ creates the source field and $T_{\text {mag }}$ represents the shielding effect of the ferromagnetic materials.

It remains now to determine the charge distribution on the external surface. We consider the machine as a whole ferromagnetic cylinder with an internal field source created by the distribution $T$ described previously (its influence can be expressed with a simple numerical integration on the air gap surface). Then, we can use a classical global integral equation to obtain external charges $q$ (Durand, 1968):

$$
\frac{\mu_{r}+1}{\mu_{r}-1} q(P)-\frac{1}{2 \pi} \iint_{S} q(M) \frac{\overrightarrow{\mathbf{r}} \cdot \overrightarrow{\mathbf{n}}}{\mathbf{r}^{3}} \mathrm{~d} S=2\left(\overrightarrow{\mathbf{n}} \cdot \overrightarrow{\mathbf{B}}_{0}(P)\right)
$$

where $\mathbf{r}$ is the vector between a point $P$ of the surface and $M$ the integration point, $\mathbf{B}_{0}$ is the induction created by internal normal dipoles distribution $T$. By meshing the surface and using a point-matching approach, we get a full linear system resolved with LU decomposition. Then, charges distribution $q$ is obtained and the stray magnetic field can be computed.

Once the values of normal dipoles $T$ and externals charges $q$ at each time step is obtained, the stray magnetic field created in the air can be calculated thanks to a relation:

$$
\vec{B}=\frac{\mu_{0}}{4 \pi} \iint_{S_{\text {air_gap }}}\left(3 \frac{\overrightarrow{\mathbf{T}} \cdot \overrightarrow{\mathbf{r}}}{\mathbf{r}^{5}} \overrightarrow{\mathbf{r}}-\frac{\overrightarrow{\mathbf{T}}}{\mathbf{r}^{3}}\right) \mathrm{d} S+\frac{\mu_{0}}{4 \pi} \iint_{S_{\text {extemal }}} q \frac{\overrightarrow{\mathbf{r}}}{\mathbf{r}^{3}} \mathrm{~d} S
$$

As a consequence, this model is thus easy to implement by considering two cylindrical surfaces (air gap and external surfaces) meshed into rectangular elements. The air gap one is carried out with uniform normal dipoles distribution. Note that in this model, the mesh does not have any motion. The computation of the integral equations (9) and (10) is provided by Gauss numerical integration method.

One of the main advantages of this approach is the simplicity of the machine description. In particular, lots of references provide analytical expressions of the field in 
the air gap of electric machine in faulty operation modes (Timar, 1989). For example, these harmonic expressions concern rotor short-circuits and rotor dynamic eccentricity:

$$
\begin{gathered}
T(\theta, t)=k \times \cos \left(p \theta-\omega_{s} t\right)+\lambda_{\mathrm{cc}} \times \cos \left(\theta-\frac{\omega_{s} t}{p}\right) \\
T(\theta, t)=k \times \cos \left(p \theta-\omega_{s} t\right) \times\left(1+\lambda_{\mathrm{de}} \times \sin \left(\theta-\frac{\omega_{s} t}{p}\right)\right)
\end{gathered}
$$

where $\lambda_{\mathrm{cc}}$ and $\lambda_{\mathrm{de}}$ represent the constants linked to the fault amplitude of short-circuits and eccentricity, respectively.

\section{Analysis of stray magnetic field}

In this section, the models are applied to a no-load operation mode of a complex four-poles synchronous generator working at low frequency. In a first step, the stray magnetic field of the healthy machine is computed thanks to our both models and the results are compared to those obtained by our reference. This reference is a standard FEM modeling (Figure 4(a)) in Flux3D software. A post-processing magnetic moments method to compute the stray magnetic field is used. Once both models are validated, different rotor faults (short-circuit windings, dynamic eccentricity) are introduced and their influence on the stray magnetic field are analyzed.

\subsection{Modelling}

We have modeled in the first place a machine without iron housing.

The model based on homogenized FEM approach has been carried out (Figure 4(b)) in Flux3D. Different regions correspond to different permeability values and directions. To avoid the field singularity around conductors crossing the material, the rotor windings are set in the machine air gap. Because of the FE numerical noise in the air region, stray magnetic field is also calculated by a post-processing magnetic moments method. Let us notice that the number of elements is highly reduced.

We have also modeled the machine with our integral approach (Figure 4(c)). The internal cylinder is the air gap surface with normal dipoles distribution. The external surface represents the charge distribution obtained after the resolution of the linear system.

\subsection{D stray magnetic field computation}

The FEM homogenized model, the air gap surface model and the standard FEM model have been compared. The stray magnetic field obtained on the sensors outside the healthy machine (Figure 4(c)) are presented and show a very good adequacy (Figure 5) for all modeling, which enables us to validate our approaches.

In this case the error is less than 10 percent, in some other cases still better than 20 percent.

\subsection{Low-frequency analysis of stray magnetic field}

The stray magnetic field created by healthy studied machine can be approximated as follow (Wikswo and Swinney, 1985). 


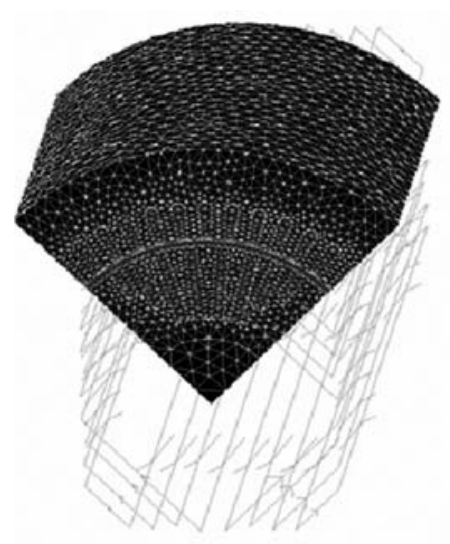

(a)

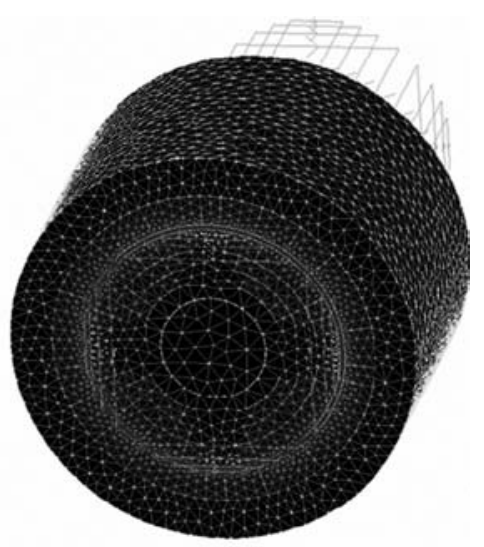

(b)
Figure 4.

(a) 3D standard FEM modeling; (b) homogenized FEM modeling of the whole machine; (c) boundary integral modeling of the whole machine
External surface with charges distribution

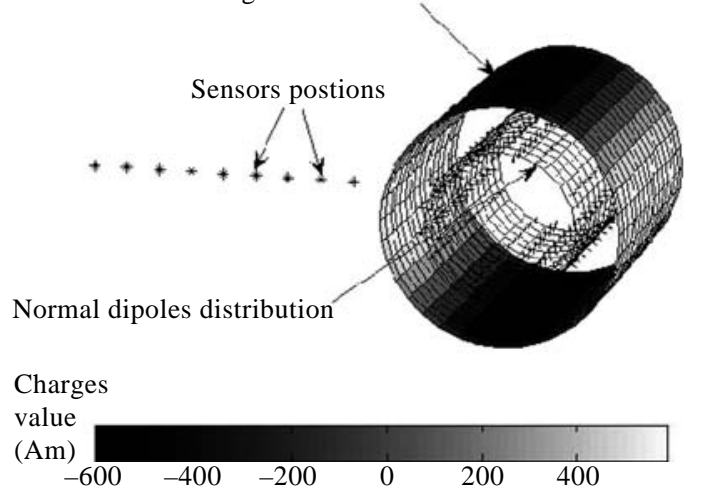

(c)

Its stray magnetic field can be approximated by the rotation of a quadrupole (four-poles electrical machine) at the rotating pulsation $\omega_{\mathrm{s}} / 2$ (Figure 6). In this case, the spectrum of this field will be constituted by a spectral line at synchronous frequency $f_{s}$.

If we introduce a rotor short-circuits of one pole machine, the machine loses its symmetry. The stray magnetic field can be approximated by the sum of two more simple representations (Figure 7):

(1) one quadrupole, representative of the healthy machine (magnetic signature in $\left.1 / r^{4}\right) ;$ and

(2) a more complex term with a very rich harmonic decomposition.

If we introduce a dynamic eccentricity in this machine, its symmetry is lost. The stray magnetic field can be simply approximated by the sum of three distributions (Figure 8): 

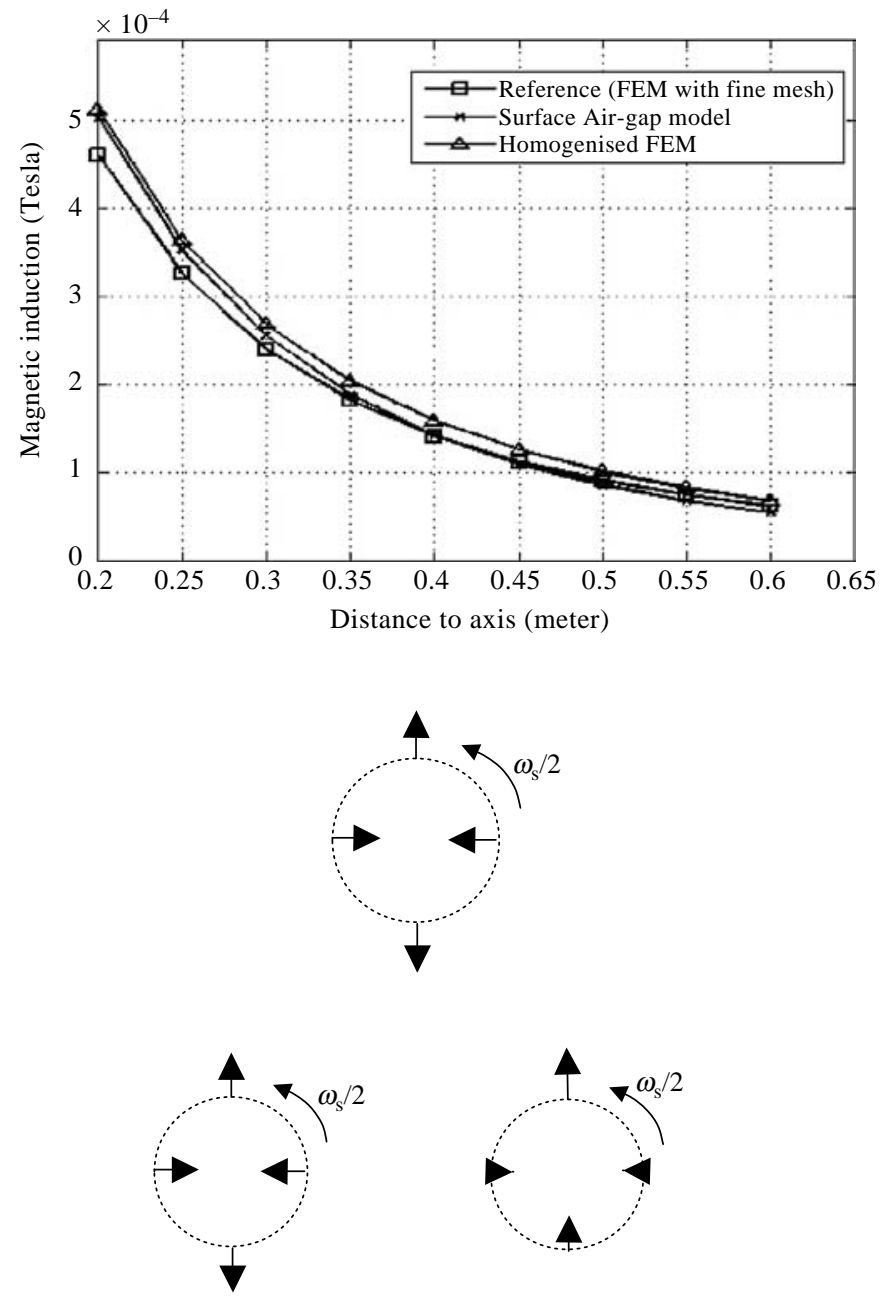

Note: This representation is the sum a quadrupole and a more complex term

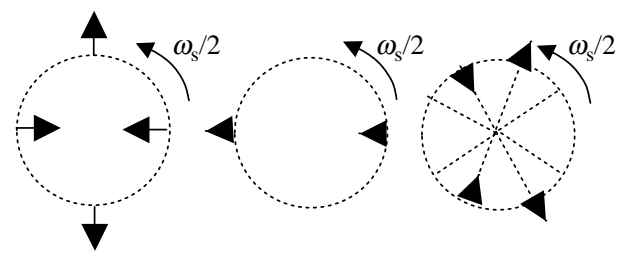

Note: This representation is the sum a dipolar term, a quadrupole one and an octopole one
Figure 5.

Comparison of stray magnetic field obtained with our models and standard FEM

Figure 6.

Representation of a healthy machine

\section{Figure 7.}

Representation with a fault in the rotor windings
Figure 8.

Representation with a rotor dynamic eccentricity 
(1) the rotation of a quadrupole, representative of the healthy machine (magnetic signature in $\left.1 / r^{4}\right)$;

(2) the rotation of two dipoles (magnetic signature in $1 / r^{3}$ ); and

(3) the rotation of an octopole (magnetic signature in $1 / r^{5}$ ) which will be largely attenuated because of its strong decreasing law.

Thanks to our models and the qualitative approximations above, faulty machines can thus be studied with an acceptable number of elements.

The first fault considered is a one-ninth rotor short-circuits of one pole. The model used is the homogenized one. By analyzing the radial component of the stray magnetic field on one magnetic sensor outside the machine, we obtain the following low-frequency spectrum (Figure 9).

Added to the synchronous frequency $\left(f_{s}=5 \mathrm{~Hz}\right)$, a new spectral component appears at the rotation speed $(2.5 \mathrm{~Hz})$.

The air gap surface model has also used to predict the magnetic stray field created by faulty machine with a dynamic eccentricity. The low-frequency spectrum is obtained.

Added to the synchronous frequency $\left(f_{s}=5 \mathrm{~Hz}\right)$ on Figure 10, two new spectral components appear at 2.5 and $7.5 \mathrm{~Hz}$.

\section{Conclusions}

In this paper, two new models are presented to compute the 3D stray magnetic field created by electric machines. The first one presents a well known homogenized technique, but originally used to compute the stray magnetic field. The second is a new, very light and easy to implement one. These two models permit also to precisely predict the frequency contents and the associated amplitudes of the stray magnetic field created by faulty machines. These models can easily be used to develop new tools methods for fault monitoring.

\section{Figure 9.}

Low frequency spectrum obtained by the

homogenized FEM model - comparison between healthy machine and rotor short-circuit machine

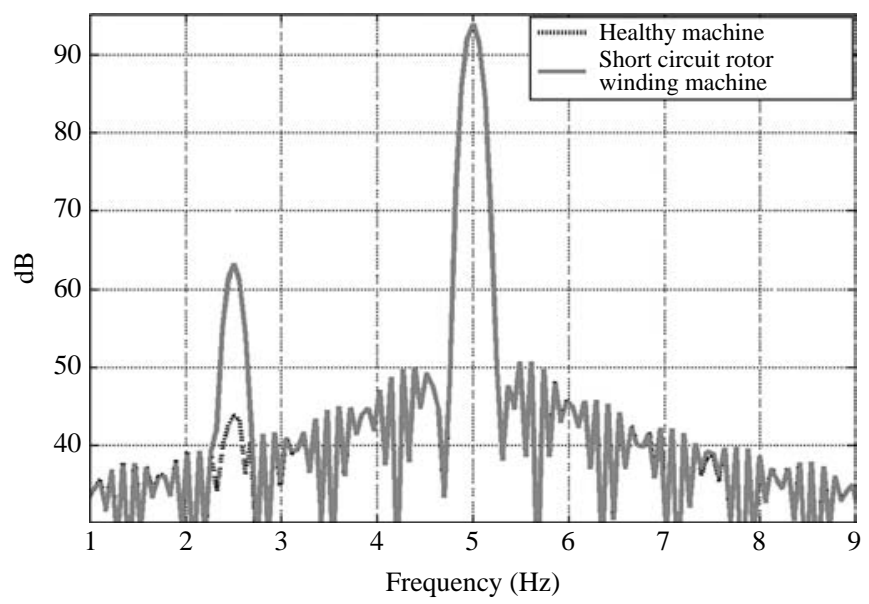




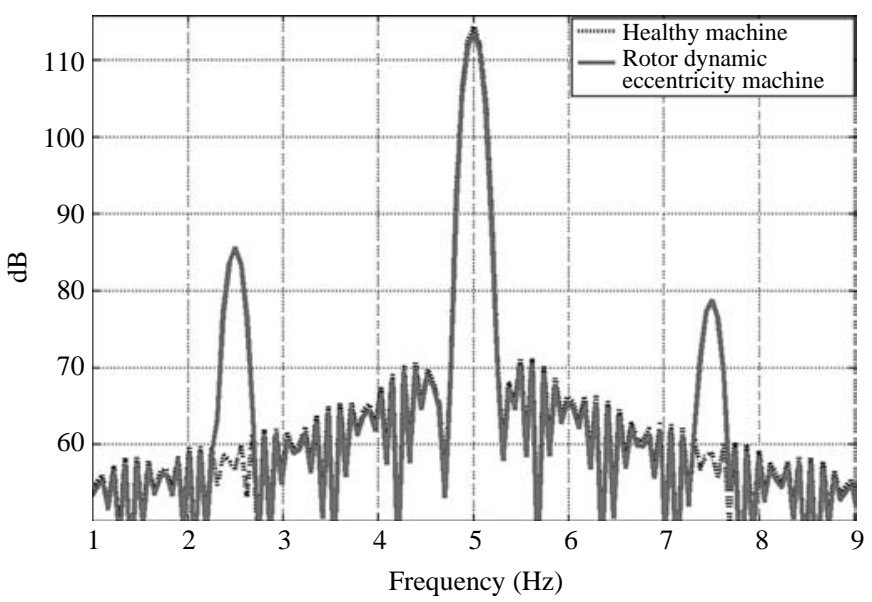

Note: Comparison between healthy machine and a machine with an eccentricity
Figure 10. Low-frequency spectrum obtained by the "air gap" surface model

\section{References}

Durand, E. (1968), Magnétostatique, Masson et Cie, Paris.

Froidurot, B., Rouve, L-L., Foggia, A., Bongiraud, J-P. and Meunier, G. (2002), "Magnetic discretion of naval propulsion machines", IEEE Trans. Magn, Vol. 38 No. 2, pp. 1185-8.

Henao, H., Demian, C. and Capolino, G-A. (2003), "A frequency-domain detection of stator winding faults in induction machine using an external flux sensor", IEEE Transactions on Industry Applications, Vol. 39 No. 5.

Selvaggi, J.P., Salon, S., Kwon, O-M. and Chari, M.V.K. (2004), "Calculating the external magnetic field from permanent magnets in permanent-magnet motors-an alternative method", IEEE Trans. Magn, Vol. 40 No. 5, pp. 3278-85.

Timar, P.L. (1989), Noise and Vibration of Electrical Machines, Elsevier Science, New York, NY.

Wikswo, J.P. Jr and Swinney, K.R. (1985), "Scalar multipole expansions and their dipole equivalents”, Journal of Applied Physics, Vol. 57 No. 9, pp. 4301-8.

\footnotetext{
About the authors

V.P. Bui was born in Danang, Vietnam, in 1980. He graduated in electrical engineering from HoChiMinh City University of Technology, Vietnam, in 2003. He is currently a PhD student at the Grenoble Electrical Engineering Laboratory (G2Elab), France. He studies the magnetic field computation methods, faults diagnosis of the electrical machines.

O. Chadebec was born in Sens, France, in 1973. He graduated in electrical engineering from the Ecole Nationale Supérieure d'Ingénieurs Electriciens de Grenoble (ENSIEG), France, in 1997. He received his $\mathrm{PhD}$ in electrical engineering in 2001 from the Institut National Polytechnique de Grenoble (INPG), France. At the present time, he is Research Associate CNRS in the team "Modeling and CAD in electromagnetism" of the Grenoble Electrical Engineering Laboratory (G2Elab), France. His current interests are magnetic field computation methods, inverse problems and low magnetic measurements. O. Chadebec is the corresponding author and can be contacted at: olivier.chadebec@leg.ensieg.inpg.fr
} 
L-L. Rouve is currently a Research Engineer at the INPG, in the team "ERT CMF" of the Grenoble Electrical Engineering Laboratory (G2Elab), France. Her current interests are low-magnetic field measurements, electrical machines, inverse problems and identification.

J-L. Coulomb was born in Nîmes, France, in 1949. He graduated in electrical engineering from the (ENSIEG), France, in 1972. He received his PhD in 1975 and the DSc in 1981 from Institut National Polytechnique de Grenoble (INPG), France. He is currently a Professor at the INPG. His activity of research is in the team "Modeling and CAD in electromagnetism" of the Grenoble Electrical Engineering Laboratory (G2Elab), France, relates to the field computation, the finite elements, the mesh generation, optimization and inverse problems, and the CAD software. 